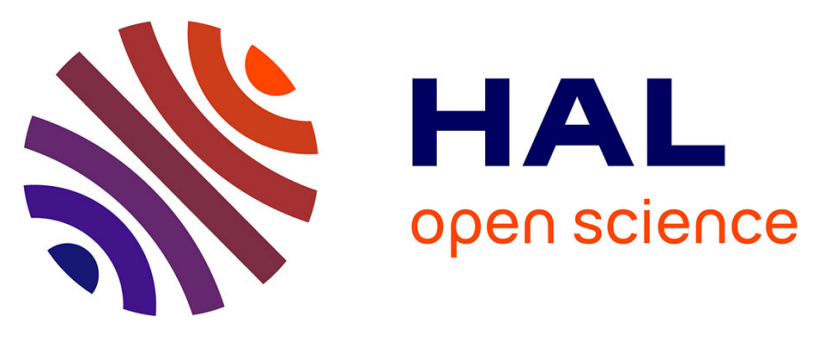

\title{
The Mayo Adhesive Probability score can help predict intra- and postoperative complications in patients undergoing laparoscopic donor nephrectomy
}

Quentin Franquet, Xavier Matillon, Nicolas Terrier, Jean-Jacques Rambeaud, Sebastien Crouzet, Jean-Alexandre Long, Hakim Fassi-Fehri, Ricardo Codas-Duarte, Delphine Poncet, Thomas Jouve, et al.

\section{To cite this version:}

Quentin Franquet, Xavier Matillon, Nicolas Terrier, Jean-Jacques Rambeaud, Sebastien Crouzet, et al.. The Mayo Adhesive Probability score can help predict intra- and postoperative complications in patients undergoing laparoscopic donor nephrectomy. World Journal of Urology, In press, 10.1007/s00345-020-03513-4 . hal-03001269

\section{HAL Id: hal-03001269 https://hal.science/hal-03001269}

Submitted on 12 Nov 2020

HAL is a multi-disciplinary open access archive for the deposit and dissemination of scientific research documents, whether they are published or not. The documents may come from teaching and research institutions in France or abroad, or from public or private research centers.
L'archive ouverte pluridisciplinaire HAL, est destinée au dépôt et à la diffusion de documents scientifiques de niveau recherche, publiés ou non, émanant des établissements d'enseignement et de recherche français ou étrangers, des laboratoires publics ou privés. 


\section{The Mayo Adhesive Probability score can help predict intra- and postoperative complications in patients undergoing laparoscopic donor nephrectomy}

Quentin Franquet ${ }^{a}$, Xavier Matillon ${ }^{b}$, Nicolas Terrier ${ }^{a}$, Jean-Jacques Rambeaud ${ }^{a}$, Sebastien Crouzet $^{b}$, Jean-Alexandre Long ${ }^{a, c}$, Hakim Fassi-Fehri ${ }^{b}$, Ricardo Codas-Duarte ${ }^{b}$, Delphine Poncet ${ }^{a}$, Thomas Jouve ${ }^{d}$, Johan Noble ${ }^{d}$, Paolo Malvezzi ${ }^{d}$, Lionel Rostaing ${ }^{d}$, Jean-Luc Descotes $^{\mathrm{a}, \mathrm{c}}$, Lionel Badet ${ }^{\mathrm{b}}$, Gaelle Fiard ${ }^{\mathrm{a}, \mathrm{c}}$

a Department of Urology and Kidney Transplantation, Grenoble Alpes University Hospital, Grenoble, France

${ }^{\mathrm{b}}$ Department of Urology and Transplantation, Hôpital Edouard Herriot, Hospices Civils de Lyon, Lyon, France

' Univ. Grenoble Alpes, CNRS, Grenoble INP, TIMC-IMAG, 38000 Grenoble, France

${ }^{d}$ Department of Nephrology, Hemodialysis, Apheresis and Kidney Transplantation, Grenoble Alpes University Hospital, Grenoble, France

\section{Corresponding author:}

Gaelle Fiard

Department of Urology and Kidney Transplantation

Grenoble Alpes University Hospital, CS 10217

38043 Grenoble Cedex 9, France

Email: GFiard@chu-grenoble.fr

Phone: +33(0)616562344

ORCID: 0000-0003-3049-5318

\section{Keywords:}

living donor, nephrectomy, laparoscopy, intraoperative complications, postoperative complications

Word count: 2118 


\section{Abstract}

Purpose: Living donor nephrectomy is a high-stake procedure involving healthy individuals, therefore every effort should be made to define each patient's individualized risk and improve potential donors' information. The aim of this study was to evaluate the interest of the Mayo Adhesive Probability (MAP) score, an imaging-based score initially designed to estimate the risk of adherent perinephric fat in partial nephrectomy, to predict intra- and postoperative complications of living donor nephrectomy.

Materials and methods: We retrospectively reviewed the imaging, clinical, and follow-up data of 452 kidney donors who underwent laparoscopic donor nephrectomy in two academic centers.

Results: Imaging and follow-up data were available for 307 kidney donors, among which 44 $(14 \%)$ had a high MAP score $(\geq 3)$. Intraoperative difficulties were encountered in 50 patients (16\%), including difficult dissection ( $n=35)$ and bleeding $(n=17)$. Conversion to open surgery was required for 13 patients (4.2\%). On multivariate analysis, a MAP score $\geq 3$ was significantly associated with the risk of intraoperative difficulty (OR $14.12(5.58-35.7), p<0.001)$ or conversion to open surgery (OR $18.96(3.42-105.14), p=0.0042)$. Postoperative complications were noted in 99 patients (32\%), including 12 patients (3.9\%) with Clavien-Dindo grade III-IV complications. On multivariate analysis, a high MAP score was also associated with the risk of postoperative complications (OR 2.55 (1.20-5.40), $p=0.01$ ).

Conclusions: In this retrospective bicentric study, a high MAP score was associated with the risk of intra- and postoperative complications of laparoscopic donor nephrectomy. The MAP score appears of interest in the living donor evaluation process to help improve donors' information and outcomes. 


\section{Introduction}

Renal transplantation is the treatment of choice for end-stage renal disease, offering the best results both in terms of quality of life and cost-effectiveness, even for older patients $[1,2]$. Kidneys obtained from living donors offer excellent results in terms of graft function and showed an improved overall graft survival compared to deceased donors, even with an increasing donor age [3-5].

The laparoscopic approach for donor nephrectomy has become the gold standard, offering reduced postoperative pain, accelerating the return to an active life, and improving the esthetic outcomes [6]. These benefits have been proven at a time when donors were preferably chosen with limited excess weight and comorbidities. Still, organ shortage increasingly leads to the consideration of older, more frequently overweight, or even obese donors. These candidates, inherently bearing more comorbidities, can be at increased risk of intraoperative difficulties or intra- and postoperative complications. Although considerable effort has been deployed to estimate the risk for the donor of developing end-stage renal disease or long-term medical adverse outcomes after kidney donation, little is known about the predictive factors of complicated surgery or postoperative course $[7,8]$.

The Mayo Adhesive Probability score (MAP score) was first designed in 2014 to predict the risk of adherent perinephric fat and intraoperative difficulties in partial nephrectomy patients [9]. The MAP score was retrospectively determined to be an independent predictor of the risk of conversion in these patients, and associated with the risk of peri- and postoperative complications in another study suggesting using a cutoff of $3[10,11]$. The potential interest of the MAP score in predicting the difficulty of laparoscopic donor 
nephrectomy was recently suggested by Cockerill et al. who showed an association of the MAP score with the total operative time [12] .

The objectives of the present study were to evaluate whether the MAP score could help predict intra- and postoperative complications in patients undergoing laparoscopic living donor nephrectomy.

Population and methods

\section{Population}

A bicentric study was conducted in two academic departments. The charts of all kidney donors operated on between January 2004 and December 2017 were retrospectively reviewed. The study was granted institutional review board approval and registered with the INDS (French institute for health data) under the number MR 2910211218. Patients were excluded in case of missing follow-up data or if they refused the use of their medical data.

\section{Imaging review and MAP score definition}

Preoperative imaging of living donors included computed tomography with intravenous contrast and non-contrast, arterial, portal, and late excretory phases.

Anatomical variations were noted. The MAP score was determined by the same observer blinded to the patients' outcome. A random sample of 20 cases was also reviewed by 2 separate readers to assess interobserver reproducibility. A cutoff of 3 was used to define a high MAP score. Details of the MAP score definition are presented in Figure 1. 

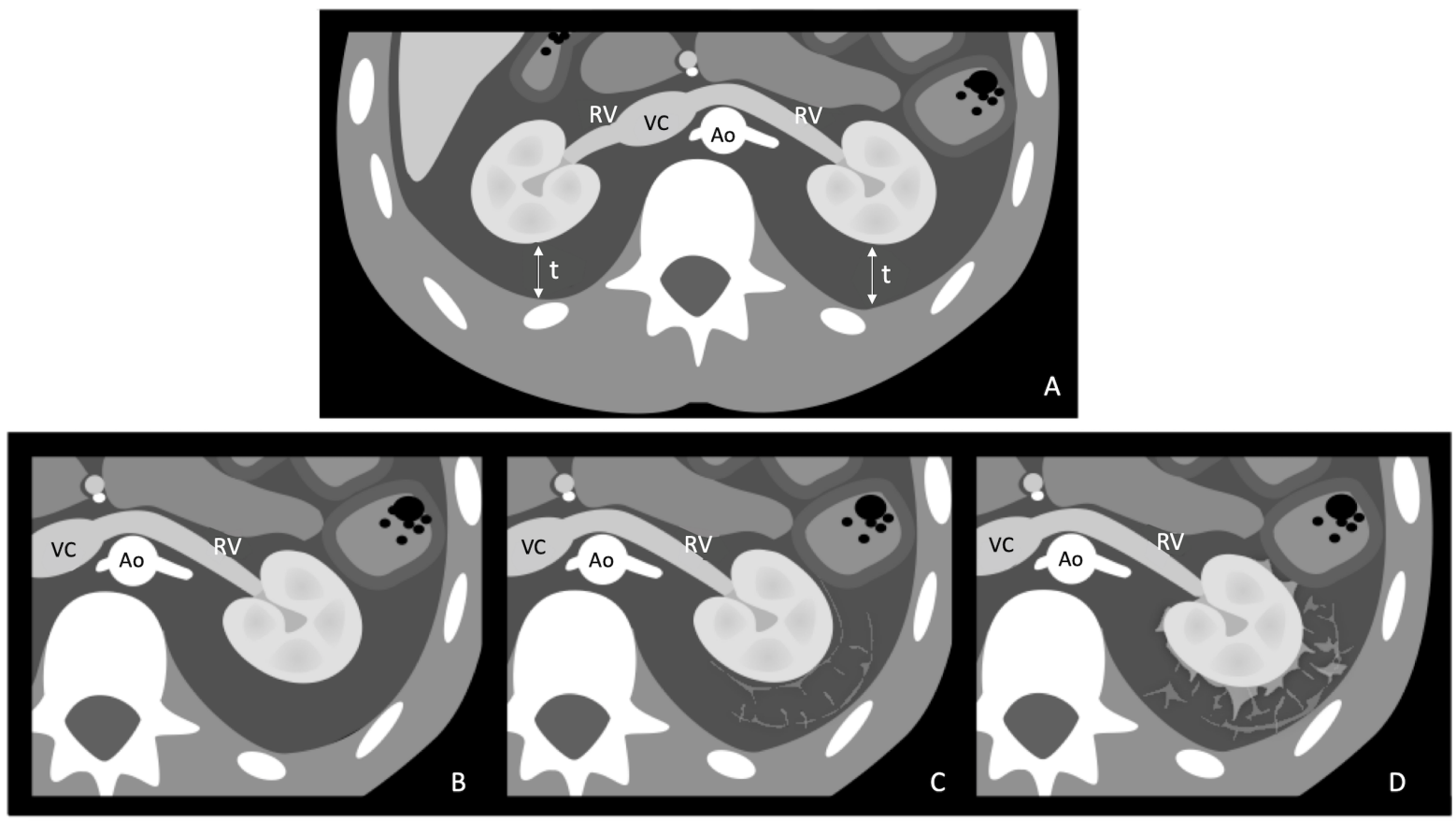

Figure 1. MAP score definition. (A) Perinephric fat thickness ( $t$ ) at the level of the renal vein. Inferior to $1 \mathrm{~cm}$ : 0 point; $1-1.9 \mathrm{~cm}: 1$ point; superior to $2 \mathrm{~cm}: 2$ points. (B-D) Perinephric fat stranding (B) absent: 0 point; (C) mild: 2 points; (D) severe stranding: 3 points ( $R V=$ renal vein, Ao=aorta, $V C=$ vena cava)

\section{Laparoscopic living donor nephrectomy technique}

All surgeries in each center were done or directly supervised by the same surgeons during the study period. Surgery was performed via a laparoscopic transperitoneal approach. A complete dissection of the renal pedicle was achieved (extra-gerotal dissection) before the kidney was dissected from its perinephric fat (intra-gerotal dissection) and placed in a laparoscopic retrieval bag. Extraction of the kidney was done through a short Pfannenstiel, lower midline or periumbilical incision, depending on past surgery history. One surgeon in center 2 developed the single-port approach after completing his learning curve in a highvolume institution.

Patients operated by an upfront open approach (sub-costal or lombotomy) were excluded. 
Vascular control was obtained using a combination of nonabsorbable Hem-o-Lok clips, metallic clips, and surgical vessel staplers. In the case of Hem-o-Lok use, a second technique was used such as a metallic clip or manual suturing of the arterial stump using a nonabsorbable uncoated blue suture monofilament polypropylene suture size 4/0 (Prolene $\left.{ }^{\mathrm{TM}}\right)$.

\section{Intra- and postoperative complications assessment}

Intraoperative complications were obtained from the operating notes and, when possible, by reviewing the video recording of the surgery. All intraoperative events disrupting the surgical plan were noted. Conversion to open surgery was defined by the need to switch from laparoscopic to open approach via a sub-coastal or laparotomy incision.

Postoperative complications (until postoperative day 30 ) were obtained from the patient's medical charts, including daily medical and nurses' notes, vital signs flow sheets, discharge, and follow-up letters. The Clavien-Dindo classification was used for complication severity rating and reporting [13].

\section{Data presentation and statistical analysis}

Data are presented as numbers (percentages) or medians (IQR). Univariate analysis was performed using Fisher's exact test for nominal variables and Student's test for continuous variables. Risk factors assessed for complications were surgical approach, gender, anatomic variation, previous abdominal surgical history, MAP score, and obesity with a BMI $\geq 30 \mathrm{~kg} / \mathrm{m} 2$. Multivariable analysis was performed using nominal logistic regression and Chisquare test or the least-squares method for nominal and continuous data, respectively. All analyses were performed using the SAS interface JMP (SAS version 14). Statistical significance was set at $p<0.05$. To account for the multiple tests performed in the comparison of the 
cohorts with or without a high MAP score, the Bonferroni correction was used. Seven tests were performed, therefore a value of $p<0.007$ was used to assess for statistical significance.

\section{Results}

\section{Study flowchart and patients' characteristics:}

The study flowchart is detailed in Figure 2. Overall, 307 donors constituted the study population, among whom 44 patients (14\%) presented with a MAP score $\geq 3$. The concordance between readers was $93 \%$ for the overall score and no case was misclassified using a cutoff of

3.

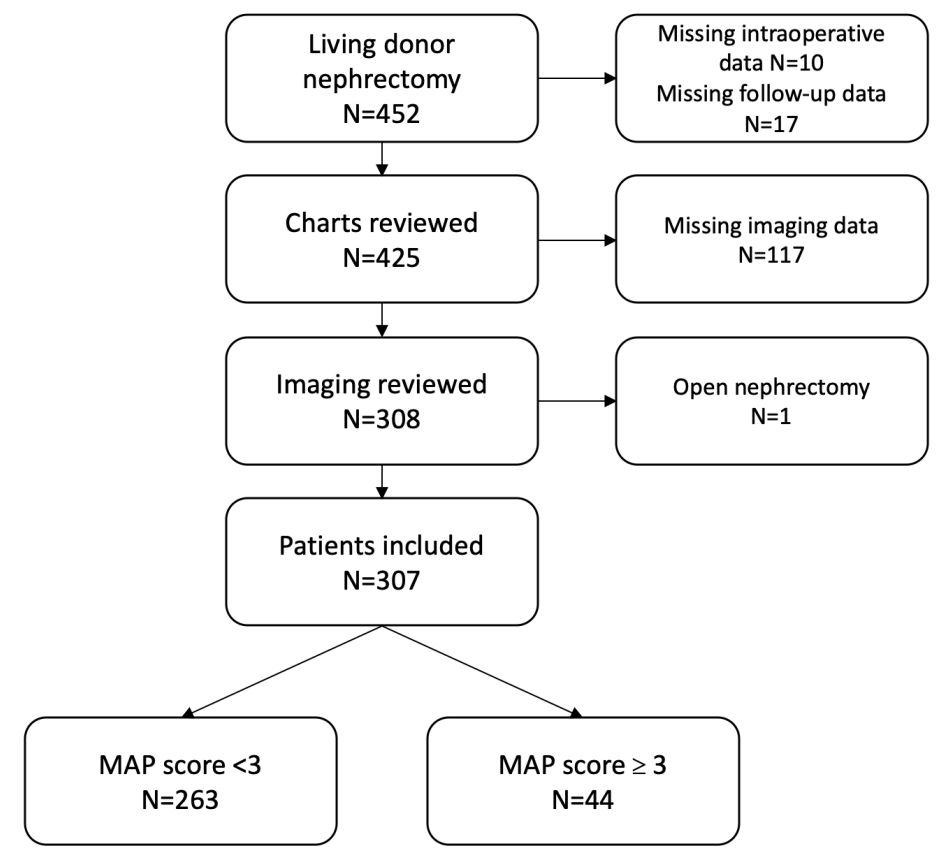

Figure 2. Study flowchart

The median age was 52 years (43-60) and the median Body Mass Index (BMI) was 24.4 $\mathrm{kg} / \mathrm{m}^{2}$ (22-27). Donors were female in $198 / 307$ (64\%) of the cases. Nearly half of the patients (49\%) had a previous history of abdominal surgery. Surgery was performed on the left side in 
284/307 cases (93\%), through a laparoscopic and single-port approach in 186 and 121 cases, respectively. An anatomical variation was described in 114 cases (37\%). The detailed patients' characteristics are described in Table 1.

\begin{tabular}{|c|c|c|c|c|}
\hline & $\begin{array}{l}\text { MAP Score }<3 \\
\qquad \mathbf{N}=\mathbf{2 6 3}\end{array}$ & $\begin{array}{c}\text { MAP score } \geq 3 \\
\qquad N=44\end{array}$ & $\begin{array}{l}\text { All patients } \\
\qquad N=307\end{array}$ & P-Value \\
\hline Age (years) - median (IQR) & $51(43-59)$ & $55(48-63)$ & $52(43-60)$ & $<0.001 *$ \\
\hline Gender - N (\%) & & & & $<0.001 *$ \\
\hline Female & $190(72)$ & $8(18)$ & $198(64)$ & \\
\hline Male & $73(28)$ & $36(82)$ & $109(36)$ & \\
\hline ASA Score - N (\%) & & & & 0.02 \\
\hline 1 & $194(74)$ & $24(55)$ & $218(71)$ & \\
\hline 2 & $69(26)$ & $19(43)$ & $88(28.7)$ & \\
\hline 3 & 0 & $1(2)$ & $1(0.3)$ & \\
\hline BMI $\left(\mathrm{kg} / \mathrm{m}^{2}\right)$ - median (IQR) & $24.1(21-26.6)$ & $26.7(25-28.6)$ & $24.4(22-27)$ & $<0.001 *$ \\
\hline $\begin{array}{l}\text { Past abdominal surgery (yes) - N } \\
\text { (\%) }\end{array}$ & $129(49)$ & $21(48)$ & 150 (49) & 0.87 \\
\hline Laterality - N (\%) & & & & 0.42 \\
\hline Right & $21(8)$ & $2(5)$ & $23(7)$ & \\
\hline Left & $242(92)$ & $42(95)$ & $284(93)$ & \\
\hline Anatomical variation - N (\%) & $91(35)$ & $23(52)$ & $114(37)$ & 0.02 \\
\hline
\end{tabular}

Table 1. Patients' characteristics (MAP score=Mayo Adhesive Probability score; ASA score=American Society of Anesthesiologists score; BMI=Body Mass Index; IQR=Interquartile Range; *statistically significant with Bonferroni correction) 
Patients with a high MAP score $(\geq 3)$ were more frequently males $(p<0.001)$, presented more frequently with obesity $(p<0.001)$. As expected, there was a strong association between a high MAP score and the description of adhesive perinephric fat during surgery $(p<0.001)$.

\section{Intraoperative complications}

Intraoperative difficulties were encountered in 50 patients (16\%), including difficult dissection ( $n=35)$ and bleeding $(n=17)$. Conversion to open surgery was required for 13 patients $(4.2 \%)$ because of inability to safely achieve planes $(n=6)$ or acute bleeding $(n=7)$. Bleeding control was performed by direct vessel control in all cases and splenectomy in one case.

On univariate analysis, a high MAP score $(\geq 3)$ and the presence of an anatomical variation were significantly associated with the risk of intraoperative difficulty. A list of all anatomical variations encountered is available as Supplementary Table. There was no significant difference in the rate of intraoperative difficulties when comparing the various surgical approaches. However, the operative time was significantly increased with the single port approach (225 minutes (200-252) vs 155 minutes (125-175), $p<0.0001)$. Obesity, male gender, and a past history of abdominal surgery were not associated with increased intraoperative difficulty. On multivariate analysis, a high MAP score $(\geq 3)$ and the presence of an anatomical variation were still significantly associated with the risk of intraoperative difficulty (Table 2).

Regarding the risk of conversion to open surgery, a high MAP score $(\geq 3)$ was the only significant risk factor in multivariate analysis (Table $\mathbf{3}$ ). 


\begin{tabular}{|l|l|l|l|}
\hline Risk factor & Odds Ratio & $\mathbf{9 5 \%} \mathbf{C l}$ & P-Value \\
\hline MAP Score $\geq \mathbf{3}$ & $\mathbf{1 4 . 1 2}$ & $\mathbf{5 . 5 8 - 3 5 . 7}$ & $<\mathbf{0 . 0 0 1 ^ { * }}$ \\
\hline Male gender & 1.54 & $0.65-3.66$ & 0.31 \\
\hline Anatomical variation & $\mathbf{2 . 2 5}$ & $\mathbf{1 . 1 1 - 4 . 5 3}$ & $\mathbf{0 . 0 2 *}$ \\
\hline Surgical approach & 0.92 & $0.43-1.97$ & 0.19 \\
\hline Past abdominal surgery & 1.01 & $0.49-2.04$ & 0.98 \\
\hline BMI $\geq 30 \mathrm{~kg} / \mathrm{m}^{2}$ & 2.15 & $0.53-8.73$ & 0.26 \\
\hline
\end{tabular}

Table 2. Risk factors of intraoperative difficulty on multivariate analysis. ( $\mathrm{Cl}=\mathrm{Confidence}$ Interval; MAP score=Mayo Adhesive Probability score; BMI=Body Mass Index; *statistically significant)

\begin{tabular}{|l|l|l|l|}
\hline Risk factor & Odds Ratio & $\mathbf{9 5 \%} \mathbf{C l}$ & P-Value \\
\hline MAP Score $\geq \mathbf{3}$ & $\mathbf{1 8 . 9 6}$ & $\mathbf{3 . 4 2 - 1 0 5 . 1 4}$ & $\mathbf{0 . 0 0 4 2}$ \\
\hline Male gender & 5.54 & $0.87-35.23$ & 0.05 \\
\hline Anatomical variation & 2.86 & $0.73-11,14$ & 0.12 \\
\hline Surgical approach & 0.31 & $0.05-1.67$ & 0.25 \\
\hline Past abdominal surgery & & & \\
\hline BMI $\geq 30 \mathrm{~kg} / \mathrm{m}^{2}$ & 1.37 & $0.38-4.92$ & 0.62 \\
\hline & 3.18 & $0.28-35.26$ & 0.29 \\
\hline
\end{tabular}

Table 3. Risk factors of conversion to open surgery on multivariate analysis. ( $\mathrm{Cl}=$ Confidence Interval; MAP score=Mayo Adhesive Probability score; BMI=Body Mass Index; *statistically significant) 


\section{Postoperative complications}

A total of 112 complications were noted in 99 donors (32\%). Most complications ( $n=106)$ were classified as minor according to the Clavien-Dindo classification (Clavien I and II). Local wound complications accounted for half of all complications $(n=49)$, including wound abscess $(n=30)$, hematoma $(n=9)$ and delayed wound healing $(n=10)$. One thrombo-embolic complication occurred (pulmonary embolism).

Twelve donors (3.9\%) presented with a severe complication requiring surgery (Clavien IIIa and IIIb) including one acute hemorrhage while the patient was in recovery, requiring emergency laparotomy (Clavien IV). No donor died.

Four donors underwent drain placement under local anesthesia for the drainage of chylous ascites. One small bowel obstruction due to internal hernia motivated iterative surgery without bowel resection. Two donors developed pleural complications (pneumothorax, pleural effusion) requiring drainage.

On multivariate analysis, a high MAP score $(\geq 3)$ was the only significant factor associated with an increased risk of postoperative complications (Table 4).

\begin{tabular}{|l|c|c|c|}
\hline Risk factor & Odds Ratio & $\mathbf{9 5 \%} \mathbf{C l}$ & P-Value \\
\hline MAP Score $\geq \mathbf{3}$ & $\mathbf{2 . 5 5}$ & $\mathbf{1 . 2 0 - 5 . 4 0}$ & $\mathbf{0 . 0 1 *}$ \\
\hline Male gender & 1.37 & $0.76-2.48$ & 0.28 \\
\hline Anatomical variation & 1.31 & $0.79-2.18$ & 0.29 \\
\hline Surgical approach & 1.96 & $1.31-3.95$ & 0.07 \\
\hline Past abdominal surgery & 1.24 & $0.74-2.06$ & 0.40 \\
\hline BMI $\geq 30 \mathrm{~kg} / \mathrm{m}^{2}$ & 1.32 & $0.52-3.30$ & 0.55 \\
\hline
\end{tabular}


Table 4. Risk factors of postoperative complications on multivariate analysis. ( $\mathrm{Cl}=$ Confidence Interval; MAP score=Mayo Adhesive Probability score; BMI=Body Mass Index; *statistically significant)

\section{Discussion}

In a background of a worldwide organ shortage, the performance of laparoscopic donor nephrectomy was found to increase the number of kidney donations [14]. However, while donor selection is being less restrictive to include donors with more comorbidities and higher $\mathrm{BMI}$, the safety of a procedure performed on healthy individuals should remain our main concern. Still, while a considerable effort has been deployed to assess the operative risk of patients undergoing partial nephrectomy for renal cancer, little has been done in the specific field of kidney donation $[15,16]$. We show here, using data gathered from two academic centers, that the Mayo Adhesive Probability score (MAP score) with a cutoff of 3, can be used to estimate the risk of intraoperative difficulty, conversion to open surgery, and postoperative complications.

Contrary to partial nephrectomy, the surgical technique for living donor nephrectomy can be adjusted to allow for a completely extra-gerotal dissection in the presence of adherent perinephric fat. Nevertheless, these results support an increased surgical difficulty, due to a more difficult mobilization of the kidney for safe vascular control, dissection of the upper pole and separation from the adrenal gland, and graft extraction.

Other studies have recently reported that donors with a higher BMI or a male gender were at higher risk of postoperative complications [17]. Still, we are now in an era of 
anticipation, where preoperative imaging gives us insight into the precise patient-specific anatomy and a significant edge when dealing with a complicated case. It is therefore not surprising that imaging-derived features such as anatomical variations or the MAP score were found to be associated with intraoperative difficulties and the risk of conversion to open surgery. This highlights the importance of obtaining high-quality preoperative imaging and its interpretation by trained radiologists.

Nevertheless, it can be opposed that even in the presence of preoperative risk factors, it may be decided to pursue with the donation plan because the benefits expected outweigh the hypothetical risks. This is probably true. However, improving patient information and consent, and raising the level of awareness of the surgical team to a potential risk of conversion or postoperative complications may prove critical to improving donors' management, safety, and outcomes. Minor complications are not without socio-economic consequences when prolonging the length of stay or sick leave of the donor.

Several studies and meta-analysis focusing on intra- and postoperative complications after kidney donation reported variable minor complication rates, probably due to inconstant reporting [18]. We describe a rate of major complications of $4 \%$, comparable to previously published data $[19,20]$. The conversion rate in our study $(4.2 \%)$ seems to be on the high side of usually reported conversion rates (around 2\%) [21]. However, reporting bias is not unlikely as Rowley et al., based on a series of 1592 contemporary laparoscopic procedures, described a quite similar conversion rate of $4.6 \%$ [22].

We must acknowledge several limitations of the present study. Its retrospective design explains a significant risk of unreported events, especially for the post-operative period. Complications and conversions were rare events, explaining the width of the confidence intervals around the estimated odds-ratios. The use of 2 techniques, conventional 
laparoscopic and single-port approaches, probably limits the reproducibility of our results. Given both teams' limited experience, no patient was operated using a retroperitoneoscopic approach, although it could have been an interesting option for patients with previous abdominal surgeries. Similarly, most donors included in this study were of white Caucasian origin and the results may not be reproduced in a population with increased racial diversity. Still, we believe that the interest of the findings obtained from "real-life" data, and the ease of implementation of the MAP score in daily practice will trigger future studies aiming at confirming these results and evaluating the impact of the score on donors' outcomes. The prospective KLIDE (Kidney Living Donor Evaluation) cohort is currently being recruited in our centers.

\section{Conclusion}

In this retrospective, bicentric study conducted among two academic departments, a high MAP score in multivariate analysis was associated with an increased risk of intraoperative difficulty, conversion to open surgery, and postoperative complications. Although the benefits of living donation may outweigh the risks and a high score does not mean that the donation project should be abandoned, these results can be used to improve donors' information and possibly safety by increasing the level of awareness of the surgical team. External validation and further prospective evaluation are mandated to confirm these results.

\section{Declarations}

\section{Funding}

Gaelle Fiard receives funding from the Fondation de France and the European Urology Scholarship Program

The authors declare that they have no conflict of interest. 


\section{Ethics approval}

This retrospective study conducted from medical charts used data obtained during routine care. Study was registered under data management reference methodology number MR

2910211218.

\section{Consent to participate}

Not applicable

\section{Consent for publication}

Not applicable

Availability of data and material

De-identified data can be made readily available to reviewers upon request

Code availability

Statistical software can be made readily available to reviewers upon request

\section{Authors' contributions}

Q Franquet: Protocol/project development, Data collection or management, Data analysis, Manuscript writing/editing

$X$ Matillon: Data collection or management, Manuscript writing/editing

N Terrier: Protocol/project development, Manuscript writing/editing

JJ Rambeaud: Protocol/project development

$S$ Crouzet: Manuscript writing/editing

JA Long: Data analysis, Manuscript writing/editing

H Fassi-Fehri: Data collection or management

$R$ Codas-Duarte: Data collection or management

D Poncet: Data collection or management

T Jouve: Manuscript writing/editing

J Noble: Manuscript writing/editing

P Malvezzi: Manuscript writing/editing

L Rostaing: Manuscript writing/editing

JL Descotes: Protocol/project development

L Badet: Protocol/project development

G Fiard: Protocol/project development, Manuscript writing/editing 


\section{References}

1. Axelrod DA, Schnitzler MA, Xiao H, et al (2018) An economic assessment of contemporary kidney transplant practice. American Journal of Transplantation 18:11681176. https://doi.org/10.1111/ajt.14702

2. Heldal K, Midtvedt K, Lønning K, et al (2019) Kidney transplantation: an attractive and cost-effective alternative for older patients? A cost-utility study. Clin Kidney J 12:888-894. https://doi.org/10.1093/ckj/sfz018

3. Yohanna S, Naylor KL, McArthur E, et al (2020) A Propensity Score-weighted Comparison of Outcomes Between Living and Standard Criteria Deceased Donor Kidney Transplant Recipients. Transplantation. https://doi.org/10.1097/TP.0000000000003337 4. Ahmadpoor P, Seifi B, Zoghy Z, et al (2020) Time-Varying Covariates and Risk Factors for Graft Loss in Kidney Transplantation. Transplant Proc. https://doi.org/10.1016/j.transproceed.2020.06.015

5. Englum BR, Schechter MA, Irish WD, et al (2015) Outcomes in kidney transplant recipients from older living donors. Transplantation 99:309-315.

https://doi.org/10.1097/TP.0000000000000607

6. Andersen MH, Mathisen L, Oyen O, et al (2006) Postoperative pain and convalescence in living kidney donors-laparoscopic versus open donor nephrectomy: a randomized study. Am J Transplant 6:1438-1443. https://doi.org/10.1111/j.1600-

6143.2006.01301.x

7. Grams ME, Sang Y, Levey AS, et al (2016) Kidney-Failure Risk Projection for the Living Kidney-Donor Candidate. N Engl J Med 374:411-421. https://doi.org/10.1056/NEJMoa1510491

8. Janki S, Dehghan A, van de Wetering J, et al (2020) Long-term prognosis after kidney donation: a propensity score matched comparison of living donors and non-donors from two population cohorts. Eur J Epidemiol 35:699-707. https://doi.org/10.1007/s10654-02000647-y

9. Davidiuk AJ, Parker AS, Thomas CS, et al (2014) Mayo adhesive probability score: an accurate image-based scoring system to predict adherent perinephric fat in partial nephrectomy. Eur Urol 66:1165-1171. https://doi.org/10.1016/j.eururo.2014.08.054 10. Khene Z-E, Peyronnet B, Kocher NJ, et al (2018) Predicting morbidity after robotic partial nephrectomy: The effect of tumor, environment, and patient-related factors. Urol Oncol 36:338.e19-338.e26. https://doi.org/10.1016/j.urolonc.2018.04.005

11. Bier S, Aufderklamm S, Todenhöfer T, et al (2017) Prediction of Postoperative Risks in Laparoscopic Partial Nephrectomy Using RENAL, Mayo Adhesive Probability and Renal Pelvic Score. Anticancer Res 37:1369-1373. https://doi.org/10.21873/anticanres.11457

12. Cockerill KJ, Young S, T Ball C, et al (2019) The Association of the Mayo Adhesive Probability (MAP) Score With Total Operative Time in Patients Undergoing Hand-assisted Laparoscopic Donor Nephrectomy. Urology 124:142-147.

https://doi.org/10.1016/j.urology.2018.10.041

13. Clavien PA, Barkun J, de Oliveira ML, et al (2009) The Clavien-Dindo classification of surgical complications: five-year experience. Ann Surg 250:187-196.

https://doi.org/10.1097/SLA.0b013e3181b13ca2

14. Kuo PC, Johnson LB (2000) Laparoscopic donor nephrectomy increases the supply of living donor kidneys: a center-specific microeconomic analysis. Transplantation 69:22112213. https://doi.org/10.1097/00007890-200005270-00047 
15. Kutikov A, Uzzo RG (2009) The R.E.N.A.L. nephrometry score: a comprehensive standardized system for quantitating renal tumor size, location and depth. J Urol 182:844853. https://doi.org/10.1016/j.juro.2009.05.035

16. Ficarra V, Novara G, Secco S, et al (2009) Preoperative aspects and dimensions used for an anatomical (PADUA) classification of renal tumours in patients who are candidates for nephron-sparing surgery. Eur Urol 56:786-793.

https://doi.org/10.1016/j.eururo.2009.07.040

17. Sterkenburg A, Kulu Y, Mieth M, et al (2020) Long-term Surgical Outcome and Risk Factors in Living Kidney Donors. Transplant Proc. https://doi.org/10.1016/j.transproceed.2019.12.044

18. Wilson CH, Sanni A, Rix DA, Soomro NA (2011) Laparoscopic versus open nephrectomy for live kidney donors. Cochrane Database Syst Rev CD006124. https://doi.org/10.1002/14651858.CD006124.pub2

19. Mjøen G, $\varnothing$ yen O, Holdaas H, et al (2009) Morbidity and mortality in 1022 consecutive living donor nephrectomies: benefits of a living donor registry. Transplantation 88:1273-1279. https://doi.org/10.1097/TP.0b013e3181bb44fd 20. Burkhalter F, Huynh-Do U, Hadaya K, et al (2017) Early complications after living donor nephrectomy: analysis of the Swiss Organ Living Donor Health Registry. Swiss Med Wkly 147:w14497. https://doi.org/10.4414/smw.2017.14497

21. Fisher PC, Montgomery JS, Johnston WK, Wolf JS (2006) 200 consecutive hand assisted laparoscopic donor nephrectomies: evolution of operative technique and outcomes. J Urol 175:1439-1443. https://doi.org/10.1016/S0022-5347(05)00648-8

22. Rowley MW, Wolf JS (2011) Risk factors for conversion to hand assisted laparoscopy or open surgery during laparoscopic renal surgery. J Urol 185:940-944.

https://doi.org/10.1016/j.juro.2010.10.063 


\begin{tabular}{|l|c|c|}
\hline & N (\%) & $\begin{array}{c}\text { Conversion due to } \\
\text { anatomical variation } \\
\mathbf{N}(\%)\end{array}$ \\
\hline All anatomical variations & $114(37)$ & $7(6)$ \\
\hline Superior polar artery & $25(8)$ & $2(8)$ \\
\hline Early renal artery bifurcation & $20(6)$ & $1(5)$ \\
\hline Double renal vein & $20(6)$ & $2(10)$ \\
\hline Double renal artery & $17(5.5)$ & \\
\hline Inferior polar artery & $16(5)$ & $1(6.2)$ \\
\hline Retro aortic left renal vein & $15(5)$ & \\
\hline Duplex collecting system & $4(1)$ & \\
\hline Ureteropelvic junction obstruction & $2(0.6)$ & \\
\hline Accessory vena cava & $1(0.3)$ & \\
\hline Nutcracker phenomenon & $1(0.3)$ & \\
\hline Renal artery aneurism & $1(0.3)$ & $1(100)$ \\
\hline Double genital vein & $1(0.3)$ & \\
\hline Renal artery fibromuscular dysplasia & & \\
\hline & & \\
\hline & & \\
\hline & & \\
\hline
\end{tabular}

Supplementary Table. Anatomical variations and conversion rates to open surgery 